\section{Effects of TNF- $\alpha$ Inhibitors on Abdominal Adiposity in Patients with Inflammatory Rheumatic Diseases}

\section{To the Editor:}

We read with great interest the recent contribution by Hmamouchi, et al ${ }^{1}$. They reported an early and significant increase in abdominal adiposity in patients with spondyloarthritis (SpA; mainly male subjects) while receiving anti-tumor necrosis factor (TNF)- $\alpha$ treatment. The patients were evaluated in a 2-year prospective study for body composition using dual-energy X-ray absorptiometry (DEXA; Hologic QDR 2000), and specific DEXA software was used to distinguish subcutaneous adipose tissue (SAT) and visceral adipose tissue (VAT). The results showed an increase in both SAT $\left(+31.9 \mathrm{~cm}^{2}\right.$ or $\left.+24.2 \%\right)$ and VAT $\left(+29.1 \mathrm{~cm}^{2}\right.$ or $+32.4 \%$ ) at 2 years in this population, raising the question of the relationship between these changes in adipose tissue and cardiovascular (CV) risk.

This is not the first study to examine the issue of adipose tissue modifications during anti-TNF therapy. We previously evaluated the longterm consequences of anti-TNF treatment on body composition and fat distribution, together with changes in serum adipokines, in a series of 20 patients with rheumatoid arthritis (RA) or ankylosing spondylitis (AS) ${ }^{2}$. Contrary to Hmamouchi's study, which was an ancillary protocol of another study, our study was specifically designed to evaluate the changes in adipose tissue under anti-TNF agents. Body composition and fat distribution (android or abdominal region versus gynoid region) were evaluated by DEXA (Lunar iDXA, GE Healthcare). We also observed a significant gain in weight $(+1.9 \%)$, body mass index $(+2.5 \%)$, total fat mass $(+11.1 \%)$, and fat in the android region $(+18.3 \%)$ over a 2 -year period, while lean mass and gynoid masses were not modified. Indeed, we also used specific DEXA software (CoreScan) to identify where the fat tissue accumulated in the abdominal region, and our results showed a clear tendency toward a gain in visceral fat $(+24.3 \% \text { or }+185.7 \mathrm{~g})^{2,3}$. These results were observed for both patients with AS and RA. In addition, this gain in fat mass occurred as early as 6 months. In parallel, we observed no major changes in serum leptin, total adiponectin, or ghrelin, while high molecular weight adiponectin and resistin ( 2 adipokines involved in metabolic control and insulin sensitivity) decreased.

Fat accumulation in the android region refers to abdominal fat that can be subclassified into SAT and VAT. It has been demonstrated that VAT rather than SAT is associated with insulin resistance and CV events. ${ }^{4}$. Visceral adiposity is 1 component of the metabolic syndrome (MetS) that is associated with increased susceptibility to ischemic heart disease and CV events. Thus, our results, together with those reported by Hmamouchi, et $a l$, indicate that the longterm administration of anti-TNF agents in patients with AS (or $\mathrm{SpA}$ ), as well as RA, induces significant changes in fat mass that are specifically localized in the visceral region. However, we would like to stress that the method used for visceral adiposity measurements in the study by Hmamouchi, et al was validated using a more recent device version than the Hologic QDR 2000 (Hologic Discovery W, configured with software version 12.1, Hologic) and in a population of South African women ${ }^{5}$, thus calling into question the validity of the reported results. In addition, the VAT assessment method using the Hologic device gives VAT and SAT area, and not real fat mass. In our study protocol, visceral adiposity measurements were previously validated on an iDXA device through comparison with computed tomography in both male and female subjects, and we were able to better quantify this fat mass gain, which was around $185 \mathrm{~g} \pm 219.7^{2}$.

Second, a higher prevalence of MetS has been reported in patients with AS and $\mathrm{RA}^{6,7}$. Clinical studies have shown an improvement in MetS during anti-TNF treatment, suggesting a favorable cardiometabolic effect of these agents 7 . But a number of studies have described changes in lipid profile under anti-TNF treatment, with an increase in both total and low-density lipoprotein cholesterol ${ }^{8}$. However, there is substantial evidence indicating that the longterm $\mathrm{CV}$ risk is improved during anti-TNF treatment in patients with $\mathrm{RA}^{9}$. Various changes in adipokine serum levels have also been reported during anti-TNF therapy and no definite conclusion can be drawn regarding these variables and their effect on metabolic balance and thus on CV risk ${ }^{2,10}$. Collectively, the changes in adipose tissue with an accumulation in the visceral region, together with changes in adipokines involving adiponectin and other adipokines related to $\mathrm{CV}$ risk, could have deleterious consequences in patients with chronic inflammatory diseases receiving anti-TNF agents. Thus, future studies evaluating the parallel changes in abdominal (visceral) fat and adipokines are warranted to better characterize the relationship between these modifications, and the $\mathrm{CV}$ risk in patients with inflammatory rheumatic diseases such as AS and RA.

ÉRIC TOUSSIROT, MD, PhD, University Hospital of Besançon, Clinical Investigation Center for Biotherapy INSERM CIC-1431, FHU INCREASE, and Department of Rheumatology, and University of Franche Comté, Department of Therapeutics, and UPRES EA 4266 Pathogens and Inflammation, SFR FED 4234, and LabEX LipSTIC, ANR-11-LABX-0021; GILLES DUMOULIN, MD, PhD, University Hospital of Besançon, Endocrine and Metabolic Biochemistry, and University of Franche Comté, Besançon, France. Address correspondence to Prof. É. Toussirot, University Hospital of Besançon, Clinical Investigation Center for Biotherapy INSERM CIC-1431, Place St. Jacques, 25000 Besançon, France. E-mail: etoussirot@chu-besancon.fr

\section{REFERENCES}

1. Hmamouchi I, Roux C, Paternotte S, Kolta S, Dougados M, Briot $\mathrm{K}$. Early increase of abdominal adiposity in patients with spondyloarthritis receiving anti-tumor necrosis factor- $\alpha$ treatment. J Rheumatol 2014;41:1112-7.

2. Toussirot É, Mourot L, Dehecq B, Wendling D, Grandclément É, Dumoulin G, et al. TNF $\alpha$ blockade for inflammatory rheumatic diseases is associated with a significant gain in android fat mass and has varying effects on adipokines: a 2-year prospective study. Eur J Nutr 2014;53:951-61.

3. Kaul S, Rothney MP, Peters DM, Wacker WK, Davis CE, Shapiro $\mathrm{MD}$, et al. Dual-energy X-ray absorptiometry for quantification of visceral fat. Obesity 2012;20:1313-8.

4. Després JP. Body fat distribution and risk of cardiovascular disease: an update. Circulation 2012;126:1301-13.

5. Micklesfield LK, Goedecke JH, Punyanitya M, Wilson KE, Kelly TL. Dual-energy X-ray performs as well as clinical computed tomography for the measurement of visceral fat. Obesity 2012;20:1109-14.

6. Malesci D, Niglio A, Mennillo GA, Buono R, Valentini G, La Montagna G. High prevalence of metabolic syndrome in patients with ankylosing spondylitis. Clin Rheumatol 2007;26:710-4.

7. Stagakis I, Bertsias G, Karvounaris S, Kavousanaki M, Virla D, Raptopoulou A, et al. Anti-tumor necrosis factor therapy improves insulin resistance, beta cell function and insulin signaling in active rheumatoid arthritis patients with high insulin resistance. Arthritis Res Ther 2012;14:R141.

8. Choy E, Sattar N. Interpreting lipid levels in the context of high-grade inflammatory states with a focus on rheumatoid arthritis: a challenge to conventional cardiovascular risk actions. Ann Rheum Dis 2009;68:460-9.

9. Barnabe C, Martin BJ, Ghali WA. Systematic review and meta-analysis: anti-tumor necrosis factor $\alpha$ therapy and cardiovascular events in rheumatoid arthritis. Arthritis Care Res 2011;63:522-9.

10. Nagashima T, Okubo-Fornbacher H, Aoki Y, Kamata Y, Kimura H, Kamimura T, et al. Increase in plasma levels of adiponectin after administration of anti-tumor necrosis factor agents in patients with rheumatoid arthritis. J Rheumatol 2008;35:936-8.

J Rheumatol 2014;41:12; doi:10.3899/jrheum.140912 